\title{
Research Paper \\ Toxicity Effects of Intraperitoneal Injection of Biochemical Nanosilver on Cardiac Tissue Structure Following Aerobic Training in Male Wistar Rats
}

\author{
Forough Javaheri Houshi ${ }^{1 \oplus},{ }^{*}$ Asieh Abbassi-Daloii ${ }^{1} \oplus$, Ahmad Abdi $^{1}$, Seyyed Javad Ziaolhagh ${ }^{2}$
}

1. Department of Exercise Physiology, Faculty of Physical Education and Sport Sciences, Ayatollah Amoli Branch, Islamic Azad University, Amol, Iran. 2. Department of Exercise Physiology, Shahrood Branch, Islamic Azad University, Shahrood, Iran.

$\begin{gathered}\text { Use yourdevic to scan } \\ \text { and read thearticle online }\end{gathered}$
Citation Javaheri Houshi F, Abbassi-Daloii A, Abdi A, Ziaolhagh SJ. [Toxicity Effect of Intraperitoneal Injection of Chemical and
Quarterly of "The Horizon of Medical Sciences". 2019; 25(3):??.
doilths://doi.org/10.32598/hms.25.3.172

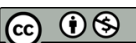

Received: 12 Nov 2018 Accepted: 12 Mar 2019 Available Online: $01 \mathrm{Jul} 2019$

Key words:

Exercise, Silver nanoparticles, Toxicity, Heart tissue, Rats

\section{ABSTRACT}

Aims Silver nanoparticles are among the most valuable products of nanoscale technology, widely used in various sciences. The present study investigated the effects of biochemical silver nanoparticles on the structure of the heart tissue of non-observatory rats in the course of aerobic training

Methods \& Materials In this experimental study, 30 male Wistar rats aged 8 to 12 weeks and weighing $34.9 \pm 202 \mathrm{~g}$ were studied. The rats were randomly divided into 6 groups of control, aerobic training, aerobic training, and nanobiological injection, aerobic and-nanochemical injection, nanobiological injection, and nanochemical injection. Chemical and biological silver was injected intraperitoneally after a period of aerobic training. The specimens were discarded after 48 hours, and the heart tissue was removed.

Findings The obtained results revealed the tissue changes, including irregularities and the convergence of chemical nanosilver group significantly increased, compared to the controls. Additionally, in the biological group, there was a slight dispersion of blood in some areas. Following the aerobic training and injection of toxic nanosilver, there was no irregularities, detachment, and hypertension. Only in some areas, sporadically, the accumulation of blood cells was observed in the aerobic training and nanochemical groups. Conclusion More tissue damage occurred in chemical silver nanoparticles, than the biological nanoparticle. Possibly, aerobic training can be highly predictive of these effects.

\section{Extended Abstract}

\section{Introduction}

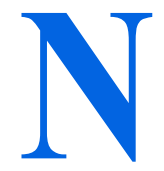

anoparticles have unique biochemical properties. These properties are not even found in the materials from which they are derived. The small size and high surface area of the nanoparticles increase their chemical activities and allow them to act as a high-performance catalyst $[1,2]$. Such increased biochemical activities of many nanoparticles have led to their widespread applications; i.e., drug delivery, vaccination, and the diagnosis or treatment of numerous diseases $[3,4]$. As the applications of silver nanoparticles increase, the odds of human exposure

* Corresponding Author:

Asieh Abbassi-Daloii, PhD.

Address: Department of Exercise Physiology, Faculty of Physical Education and Sport Sciences, Ayatollah Amoli Branch, Islamic Azad University, Amol. Iran. Tel: +98 (11) 43217126

E-mail: abbasi.daloii@gmail.com 
to these substances enhance $[8,9]$. Oxidative stress induced by oxygen free radicals is among the main pathological factors in many diseases $[18,19]$.

There have been few studies on the toxicity effects of nanosilver following an exercise program on body tissues. Vasili et al. explored the impact of aerobic exercise on hepatotoxicity induced by iron oxide nanoparticles in Wistar rats. They reported lower hepatic tissue damage and elevated liver enzymes in the low-intensity aerobic exercise group [21]. Silver nanoparticles account for $56 \%$ of the world's nanoparticles; however, few studies have been conducted on the effects of exercising on preventing the toxicity of nanoparticles on body tissues. Thus, this study aimed to evaluate the toxicity effect of chemical and biological nanosilver following an aerobic exercise course on the structure of cardiac tissue.

\section{Methods}

This experimental study was conducted on male Wistar rats at Islamic Azad University of Shahrood Branch in 2018. Thirty male Wistar rats were randomly assigned into six groups of control, Aerobic Exercise (AE), Aerobic Exercise-and NanoBiological Injection $(\mathrm{AE}+\mathrm{NB})$, Aerobic Exercise -NanoChemical Injection (AE+NCH), NanoBiological Injection (NB), and NanoChemical Injection (NCH). (Table $1 \& 2$ \& Figure 1). Chemical and biological silver nanoparticles were injected intraperitoneally at a toxic dose after a period of aerobic training [25].

\section{Results}

The obtained results suggested that tissue changes, including irregularity and coagulation types, significantly increased in the $\mathrm{NCH}$ group, compared to the controls. Moreover, in the NB group, some scattered blood droplets were observed in some areas. Following aerobic exercise and the injection of toxic nanosilver in the NB group, no irregularity, isolation, and hyperemia were observed. In AE + $\mathrm{NCH}$ group, scattered blood cell aggregation was observed in some areas.

\section{Discussion}

The study results were consistent with those of Naghsh et al. [27]. They reported a slow change in the core and fibers of cardiac muscle after injecting a toxic dose of nanosilver; possibly indicating the onset of apoptosis. In our study, structural changes in cardiac tissue, cardiac muscle cells, and hyperemia were observed in the $\mathrm{NCH}$ group.

Song et al. argued that the toxic effects of silver nanoparticles are probably due to their effects on cell viability, oxidative stress, and cell cycle. The viability of cells and their metabolism are reduced by exposure to the toxic silver nanoparticles, leading to membrane damage and the reduced activity of superoxide dismutase and glutathione peroxides [27].

Another study indicated increased apoptosis and tissue necrosis following the use of silver nanoparticles in tissues like lung and heart [28]. This impact of chemical nanosilver may be due to the use of regenerative agents for their

Table 1. Aerobic training protocol

\begin{tabular}{cccc}
\hline Week & Exercise Speed $(\mathbf{m} / \mathbf{m i n})$ & Vo2max $(\approx \%)$ & Exercise Duration (min) \\
\hline 1 & 15 & 55 & 15 \\
2 & 15 & 55 & 15 \\
\hline 3 & 20 & 70 & 20 \\
\hline 4 & 20 & 70 & 25 \\
\hline 5 & 25 & 78 & 30 \\
6 & 25 & 85 & 40 \\
7 & 30 & 85 & 50 \\
8 & 30 & 85 & 60 \\
9 & 30 & 85 & 60 \\
10 & 30 & 85 & 60 \\
\hline
\end{tabular}


Table 2. Rating changes in cardiac tissue variables

\begin{tabular}{cccccc}
\hline Group & Structural Change of Heart Tissue & Muscle Cells & Necrosis & Inflammation & Hyperemia \\
\hline Control & 0 & 0 & 0 & 0 & 0 \\
\hline $\mathrm{AE}$ & 0 & 0 & 0 & 0 & 1 \\
\hline $\mathrm{NB}$ & 1 & 0 & 0 & 0 & 2 \\
\hline $\mathrm{NCH}$ & 2 & 2 & 0 & 0 & 0 \\
\hline $\mathrm{AE}+\mathrm{NB}$ & 1 & 0 & 0 & 0 & 1 \\
\hline $\mathrm{AE}+\mathrm{NCH}$ & 2 & 1 & 0 & $\begin{array}{l}\text { Quarterly of } \\
\text { The Horizon of Medical Sciences }\end{array}$
\end{tabular}

${ }^{*}$ Changes are rated on a scale from 0 to $3 ; 0=$ No change, $1=$ Slight change, $2=$ Moderate change, $3=$ Severe change

A

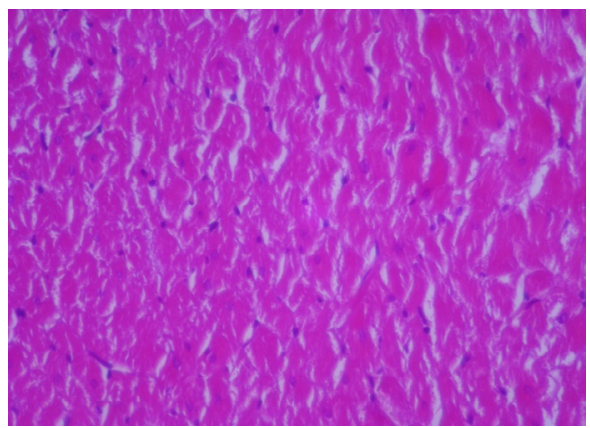

C

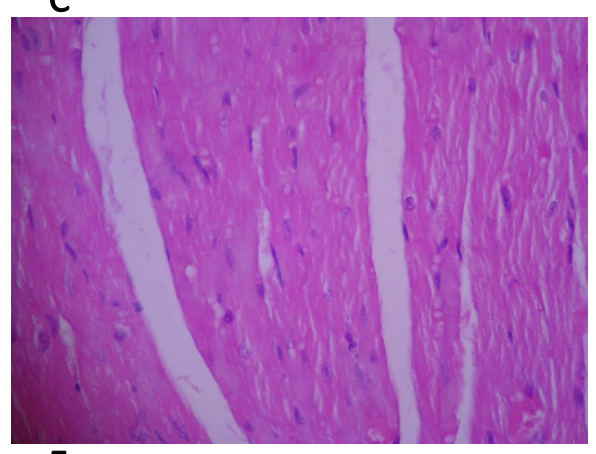

E



B

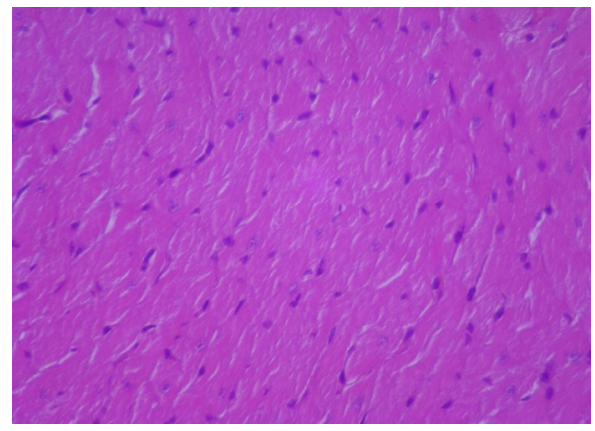

D

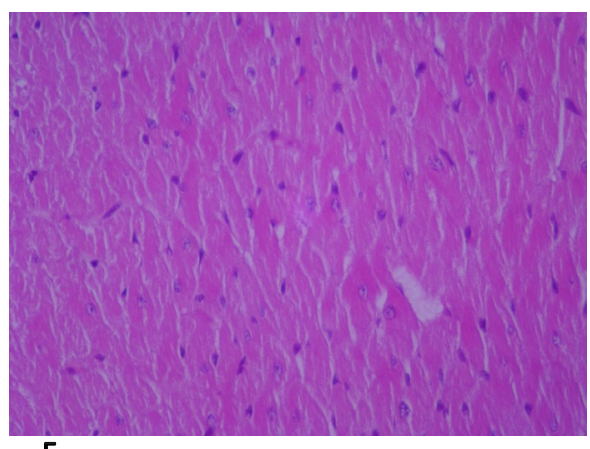

$\mathrm{F}$

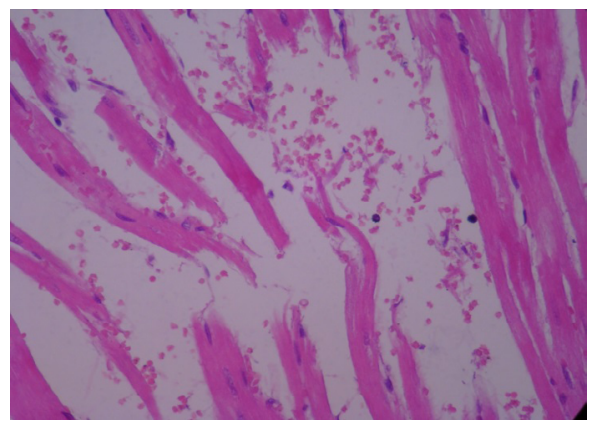

Figure 1. Histological section of the rat heart (hematoxylin \& eosin staining $\times 400$ )

Quarterly of

The Horizon of Medical Sciences

A: Control; B: AE; C: NB: D:NCH; E: AE+NB; F: AE + NCH 
products which have potential risks to human health and the environment [29].

The obtained data are also consistent with the findings of Wisløff et al. [30]. Their study on rats revealed a 15\% reduction in left ventricular hypertrophy after myocardial infarction; $15 \%$ and $12 \%$ reductions in the length and width of myocytes after aerobic exercise, respectively; and a $60 \%$ improvement in cardiac muscle contraction in people with myocardial infarction. They revealed the positive effect of aerobic exercise on heart regeneration and increased $\mathrm{Ca}^{2+}$ sensitivity on myocardial contractile [30].

It is suggested that by varying the dose of the chemical and biological silver nanoparticles, cardiac tissue structural parameters be measured after aerobic exercise. A limitation of the present study was disregarding the measurement of other structural parameters of cardiac tissue.

\section{Conclusion}

Biological and chemical nanosilver can damage the cardiac muscle tissue and cause necrosis and hyperemia in the heart tissue. Moreover, chemical silver nanoparticles appear to cause more damage to the heart tissue, compared to biological silver nanoparticles. Eventually, aerobic training can probably prevent these adverse effects to a large extent.

\section{Ethical Considerations}

\section{Compliance with ethical guidelines}

This study was performed according to the guidelines for laboratory animals of Islamic Azad University of Shahrood Branch (Code: 94.F.514.77).

\section{Funding}

This study was extracted from a Ph.D. thesis written by Forough Javaheri Houshi approved by the Department of Exercise Physiology, Faculty of Physical Education and Sport Sciences at Islamic Azad University of Ayatollah Amoli Branch.

\section{Authors' contributions}

All authors contributed in preparing this article.

\section{Conflicts of interest}

The authors declare no conflicts of interests. 
This Page Intentionally Left Blank 


\title{
بررسى اثر سميت تزريق درون صفاقي نانونقره شيميايى و بيولوزيكى بر ساختار بافت قلب، متعاقب

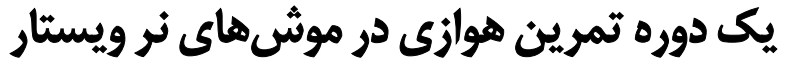

\author{
فروغ جواهرى هوشى' هـ "آسيه عباسى دلويى' ه، احمد عبدى'، سيد جواد ضيالحق' \\ ا - كروه فيزيولوزي ورزشى، دانشكده تربيتبدنى، واحد آيتالله آملى، دانشكاه آزاد اسلامى، آمل، ايران.

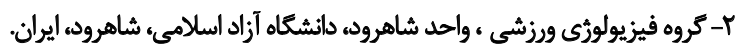

\begin{abstract}
حكيe

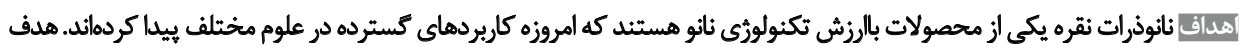

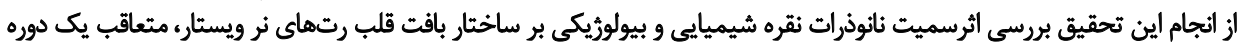
تمرين هوازي است.

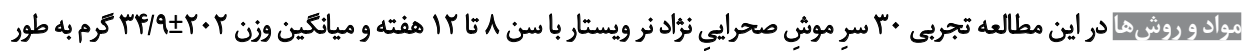



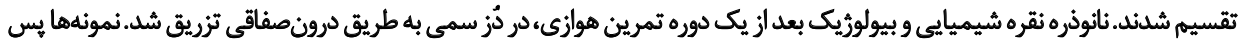

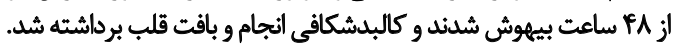

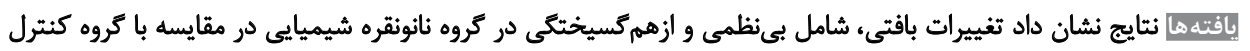

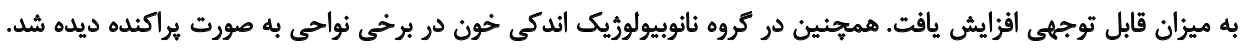

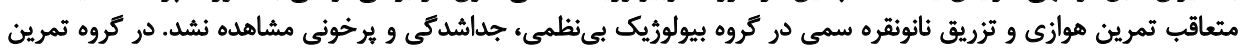

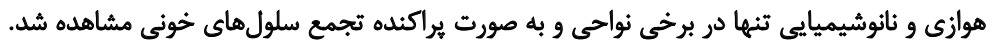

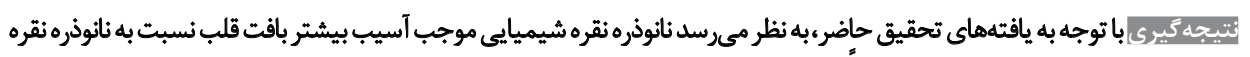

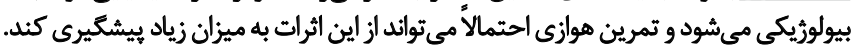

تاريخ دريافت: آب آبان Irav



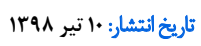

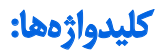

تمرين، نانوذرات نقره،

سميث، بافت قلب، تمرئ موش صحرايى

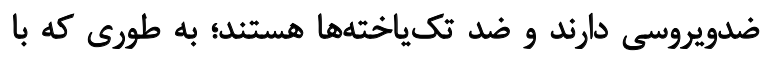

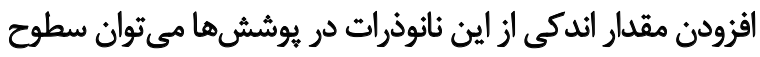

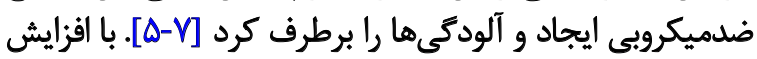

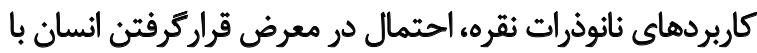

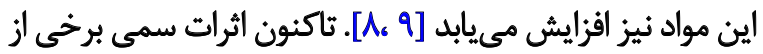

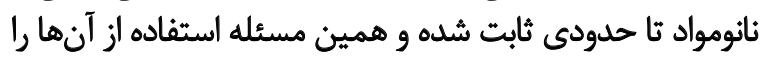

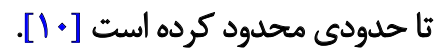

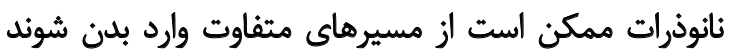

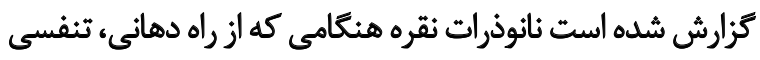

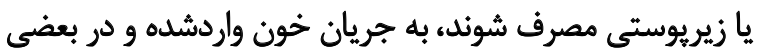

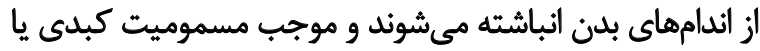

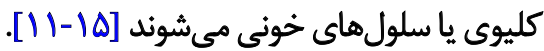

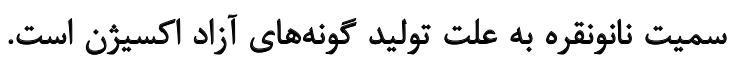



نانوذرات ويرُكى هاى فيزيكى و شيميايى منحصر بهفردى دارند؛

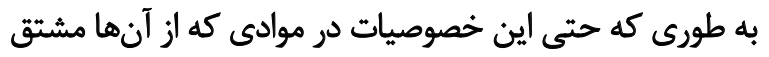

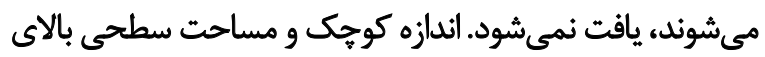

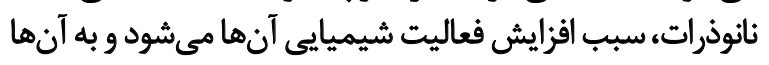

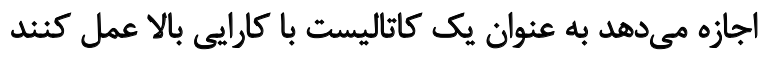

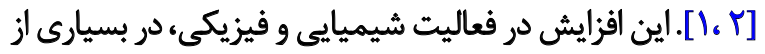

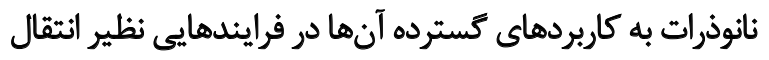

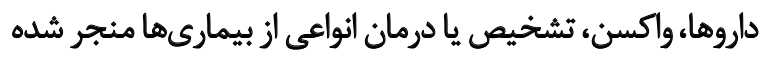

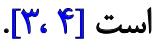

نانوذرات نقره يكى از محصولات بارزش تكنولورئ نانو هستئ

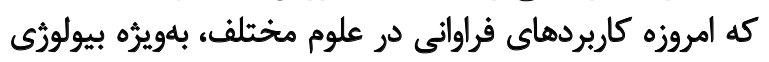



$$
\begin{aligned}
& \text { : تويسنده مسئول: } \\
& \text { دكتر آسيه عباسي دلونيفي }
\end{aligned}
$$

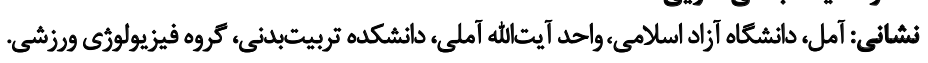



$$
\begin{aligned}
& \text { abbasi.daloii@gmail.com : يست الكترونيكى }
\end{aligned}
$$


هلاكتى و خروج فسفاتيديل سرين را نشان دادند كه با نتايج

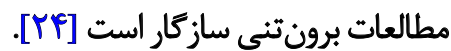
نانوذرات نقره همجيون ديكر بيوموادها، قادر به ايجاد اثرات

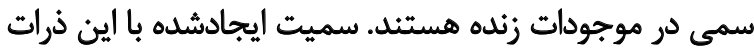

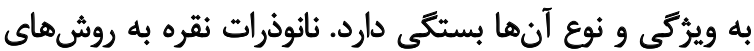

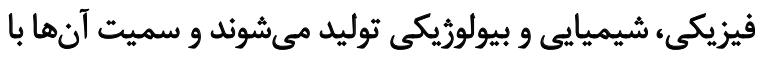

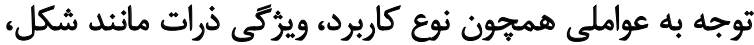

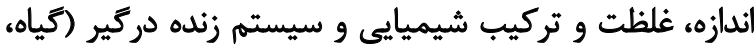

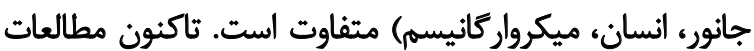

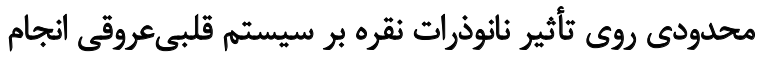

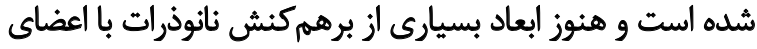
مختلف بدن مانند قلب ناشناخته باقى مانده است. بنابراين بايد به جنبه هاى ايمنى نانومواد بر سيستم قلبى إعروقي

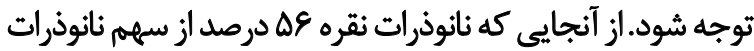

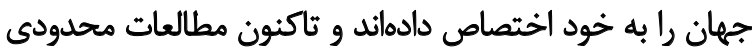

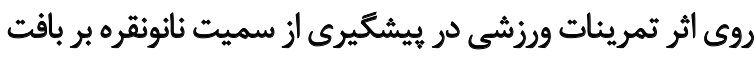

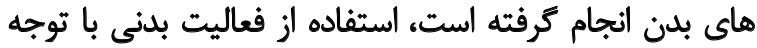

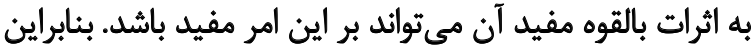

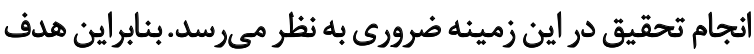

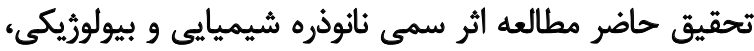
متعاقب يك دوره تمرين هوازي بر ساختار بافت قلب استي

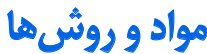

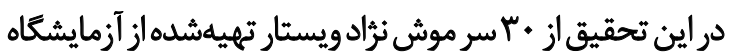

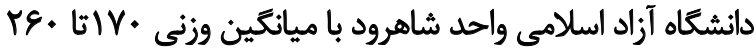
كرم كه هر هفته به طور دقيق با وزنه مخصوص

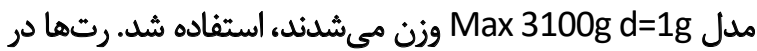

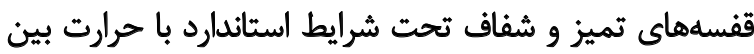

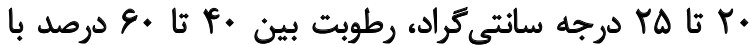

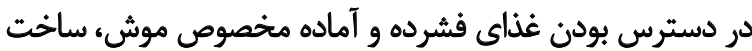

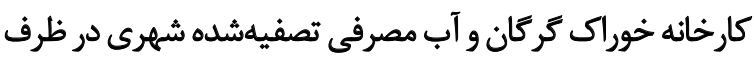

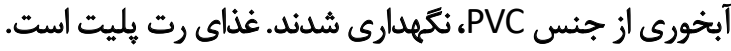
رتها به طور تصادفى به شش گروه مساوى تقسيم شدند: كروه

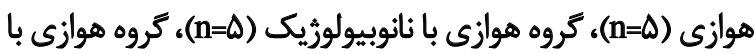

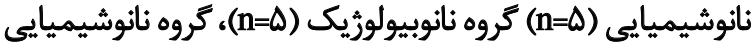



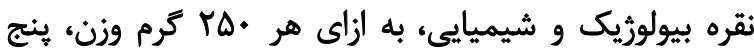

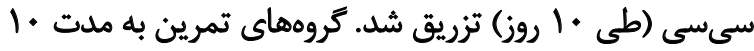





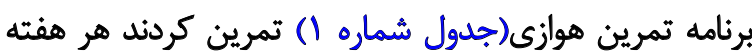

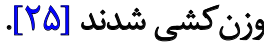

در مسيرهاى متابوليكى همه سلولهاى هوازى از اكسيرن

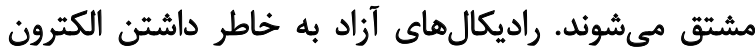

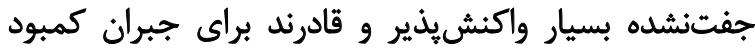

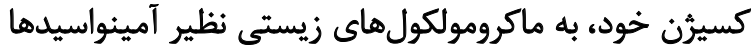

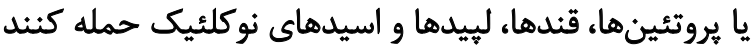
و با آسيب شديد ساختار و عملكرد سلولى، نهان نهايتاً سبب مركى

زودرس سلولى شوند [18، IV]

بلدن انسان يك سيستم دفاعي براى مقابله با راديكال هاى آزاد

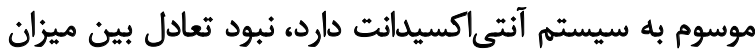



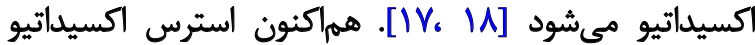

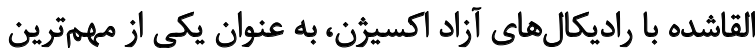

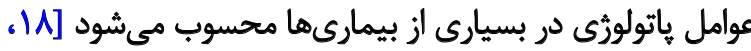

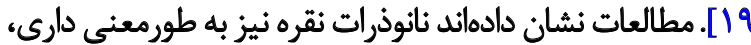

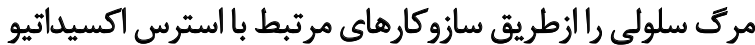

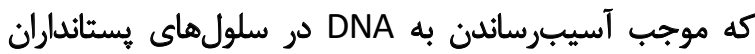

مىشود، افزايش مى مهد [ [ [1]. بسيارى از مطالعات آزمايشعاهى وابسته به دز نانوذرات نقره

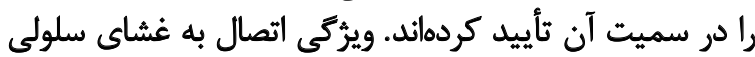



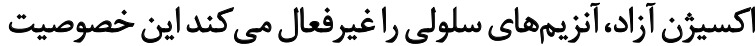

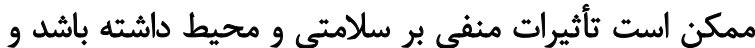

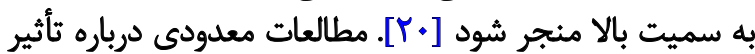

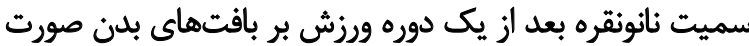





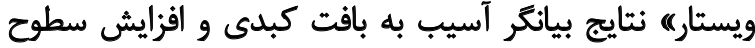

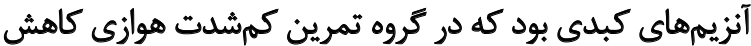

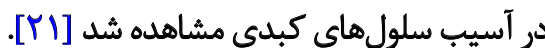

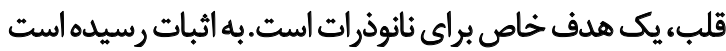



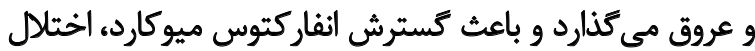

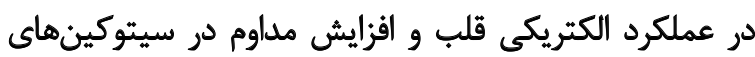

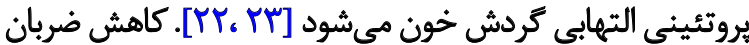



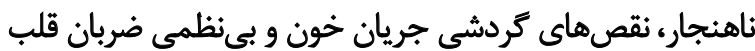
ز زمله عوارض مواجهه با ذرات نانونقره است

نتايج مطالعات حاكى از آن است كه نانوذرات نقره با اثر باثر

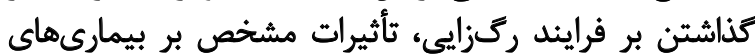






مشاهدات ميكروسـويسك و تهيه فتّوميكروكراف صورت كرفت.

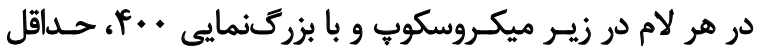

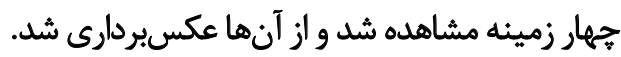

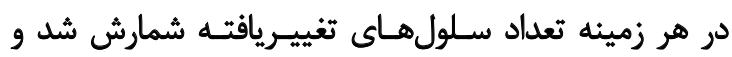

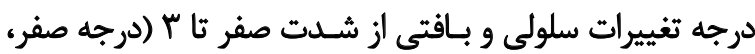

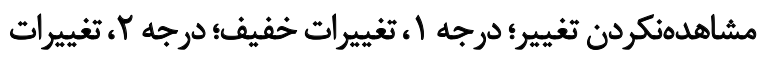

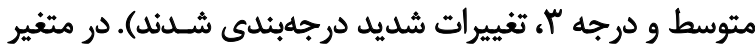

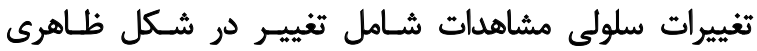


تغييرات نكروتيك (درجات مرك سلولى) بود و در متغيـر التّهاب



batio

در بررسى وزن حيوانات مطالعهشده تفاوت معنى دارى در

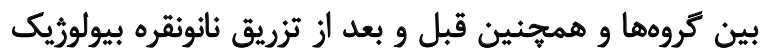

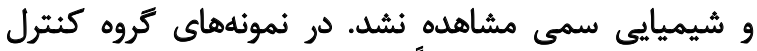

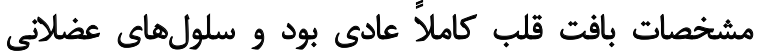

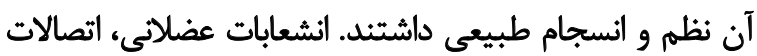

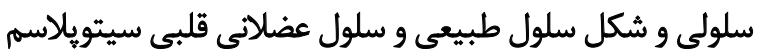

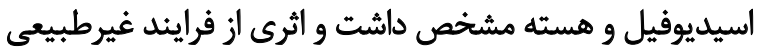



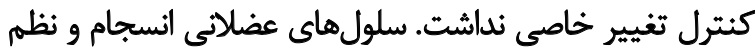

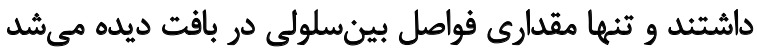

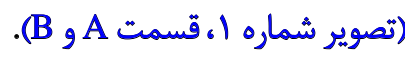

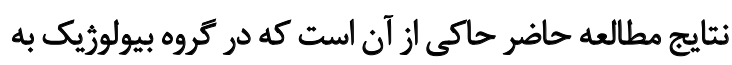

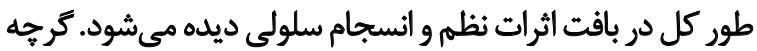

نانوذره نقره استفادهده در تحقيق حاضر از دانشكده علوم

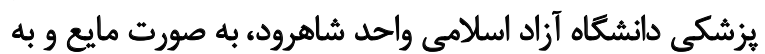

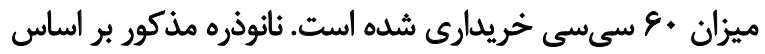

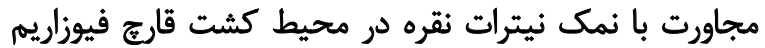

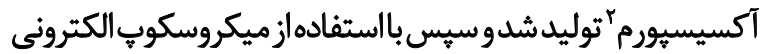

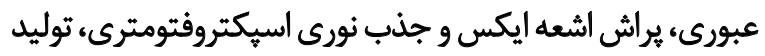

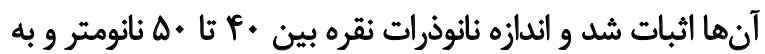

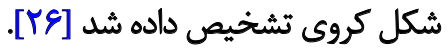
يس از انجام تمرين هوازى نانوذرات نقرهبيولوزيك و شيميايي

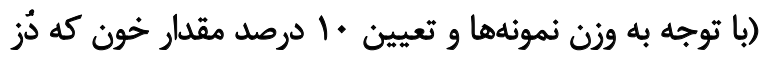

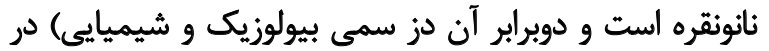

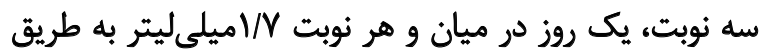

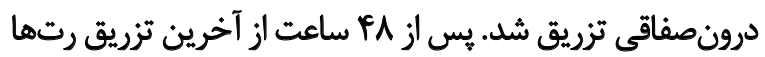

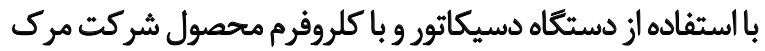

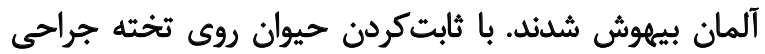

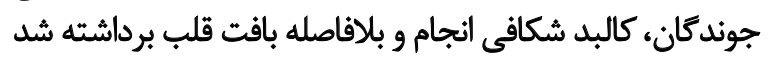

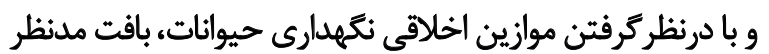

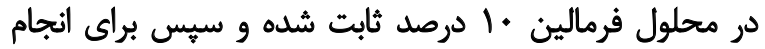
روش هاى بافتشناسي آماده شدند.

برشها با هماتوكسيلين وائوزين رنكآميزى و مطالعه شدند.

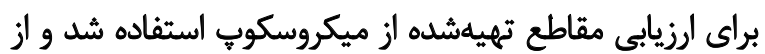

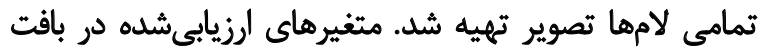

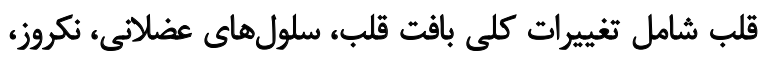

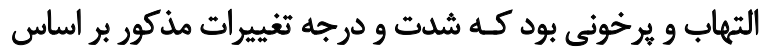

جدول ا. بروتكل تمرين هوازى

\begin{tabular}{|c|c|c|c|}
\hline مدت تمرين (دقيقه) & vرصد:vormax & سرعت تمرين (متر بر دقيقه) & هفتهاى تمرين \\
\hline 10 & $\Delta \Delta \approx$ & 10 & 1 \\
\hline 10 & $\Delta \Delta \approx$ & 10 & r \\
\hline r. & $\gamma_{0} \approx$ & $r$. & $r$ \\
\hline ro & $\gamma \cdot \approx$ & $r$. & 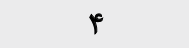 \\
\hline$r$. & $v A \approx$ & ro & $\Delta$ \\
\hline$r$. & $\Lambda \Delta \approx$ & ro & 8 \\
\hline$\Delta$. & $1 \Delta \approx$ & $\mu$. & $\gamma$ \\
\hline q. & $1 \Delta \approx$ & r. & $\Lambda$ \\
\hline q. & $\Lambda \Delta \approx$ & $r$. & 9 \\
\hline e. & $1 \Delta \approx$ & r. & 1. \\
\hline
\end{tabular}

in 
A

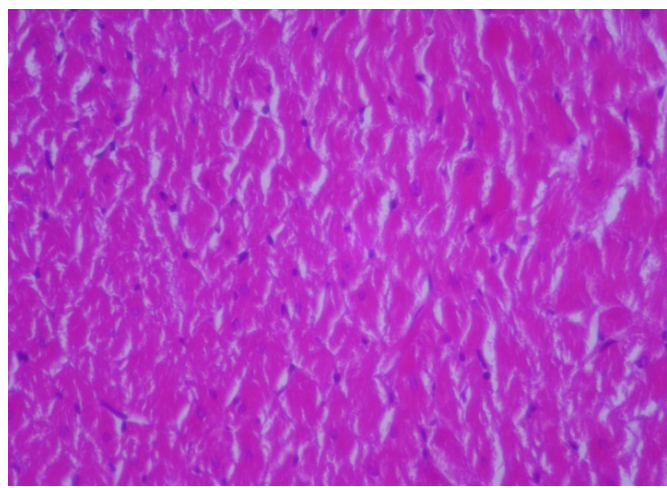

C

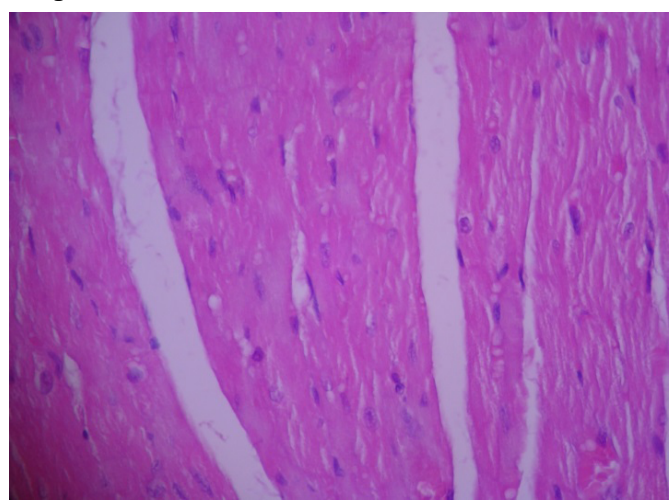

E

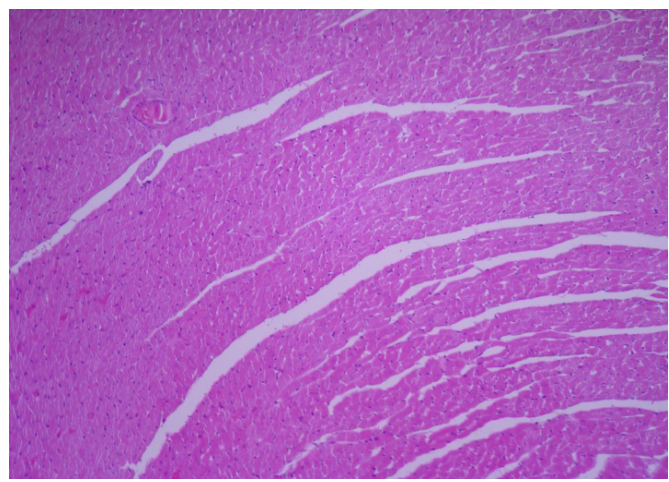

B



D

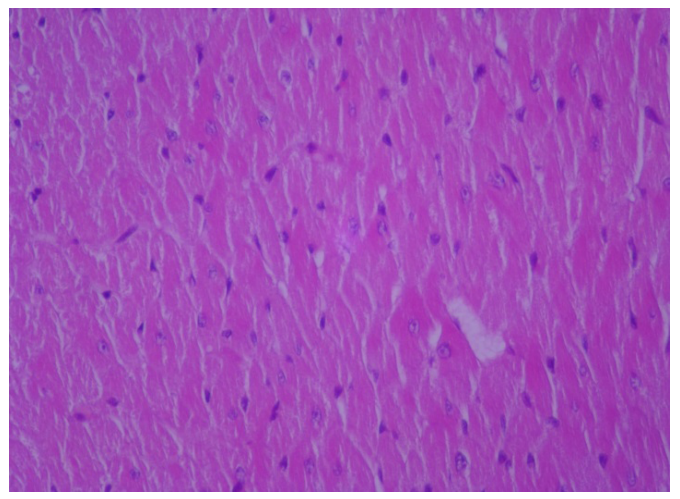

$\mathrm{F}$

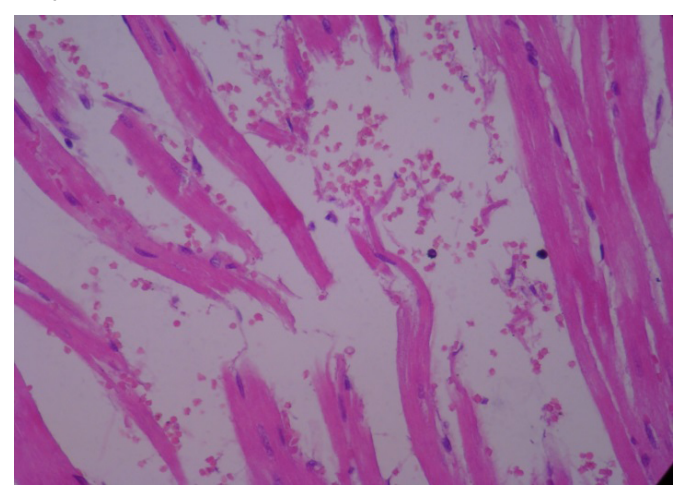

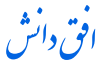

تصوير ا.مقطع بافتشناسى قلب موش صحرايى (رنكآميزى هماتوكسيلين و ائوزين××*A)

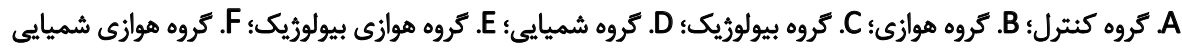

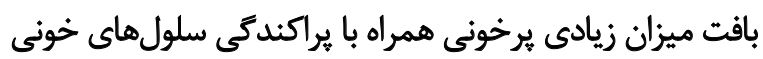

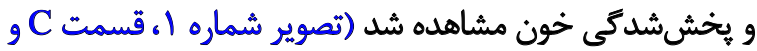

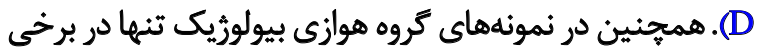

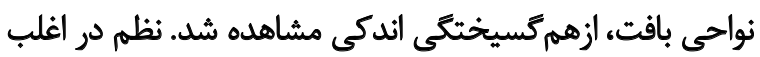

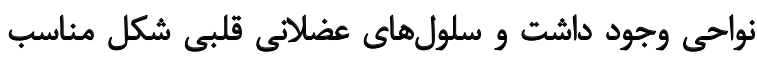



اثرى از يرخونى و التهاب در بافت مشاهده نشد. در فواصل

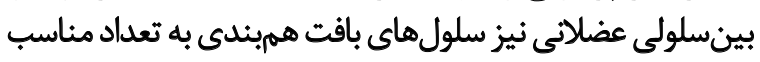

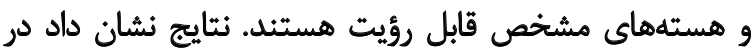

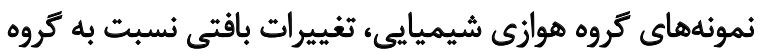

بر ميزان فواصل بينسلولى افزوده شده و جداشدكى بافتى رؤيت

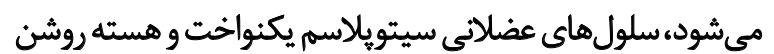

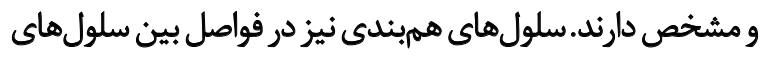

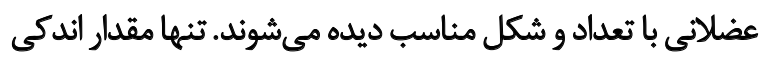

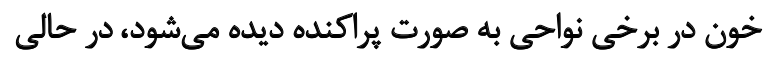

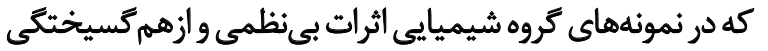
به ميزان قابل توجهى در بافت افزايش يافته استر اكرجه ستونهاى عضلات قلب شكل مناسب دارند، انشعابات

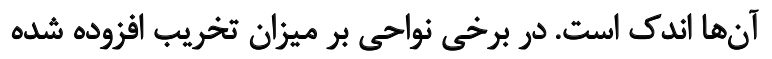

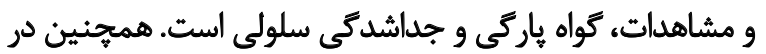


جدول r. تفكيك و درجهبندى تغييرات بديدآمده در متغيرهاى ارزيابىشده بافت قلب

\begin{tabular}{|c|c|c|c|c|c|}
\hline يرخونى & التهاب & نكروز & سلولهاى عضالى & تغيير ساختارى بافت قلب & ه \\
\hline - & • & - & - & - & 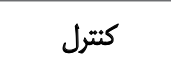 \\
\hline - & - & - & - & - & هوازى \\
\hline 1 & . & $\cdot$ & - & 1 & 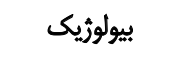 \\
\hline r & . & - & r & $r$ & 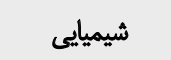 \\
\hline - & • & $\cdot$ & $\cdot$ & 1 & هوازى بيولوزيك \\
\hline 1 & . & . & 1 & r & هوازى شيميايى \\
\hline
\end{tabular}

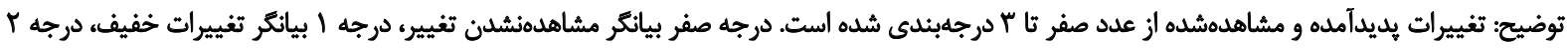

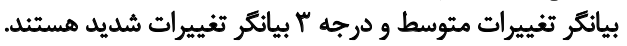

بيىنظمى وازهم كسيختكى به ميزان قابل توجهى در بافت افزايش

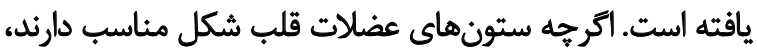

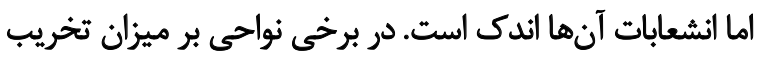

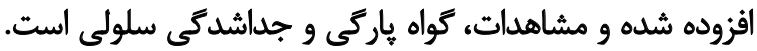

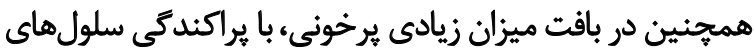

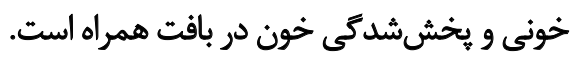

نتيجه اين مطالعه با يافتههاي نقش و همكار ان همسو است كه

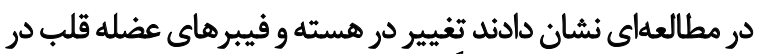

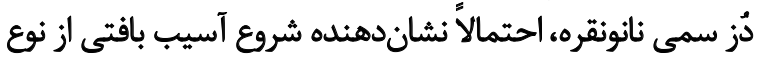

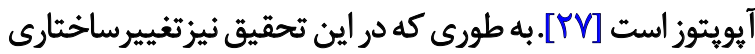

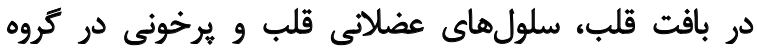

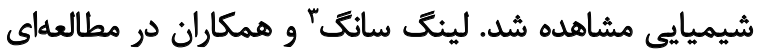

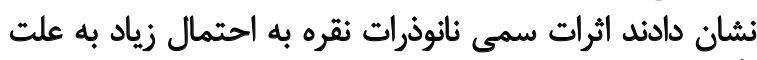

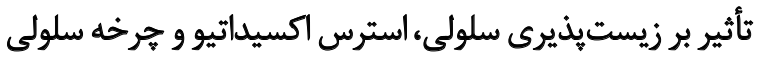

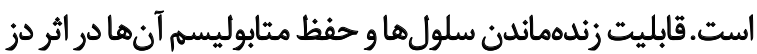

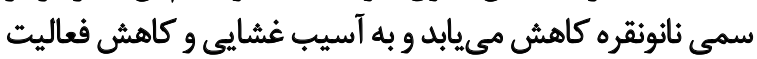

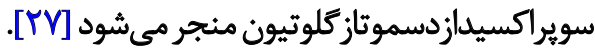

يافتههاى ديكر نيز نشاندهنده افزايش آيويتوز ونكروز بافتى

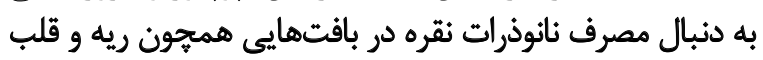

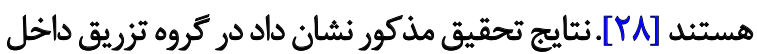



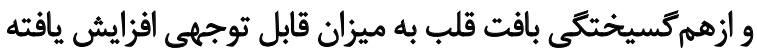

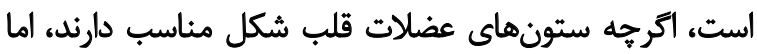

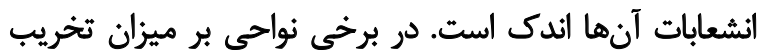

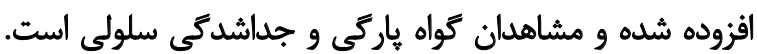

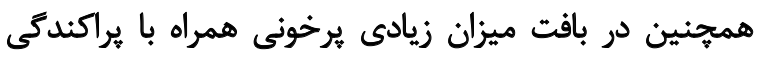

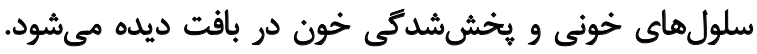

3. Ling Sang
دريافت كننده دز شيميايي، التهاب كمترى دارند؛ به طورى كه

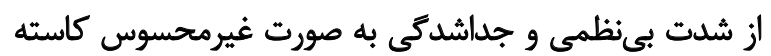

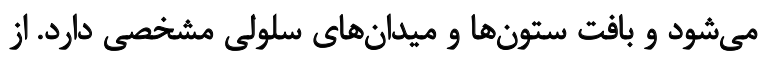

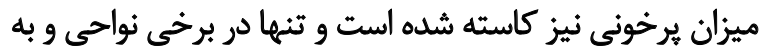

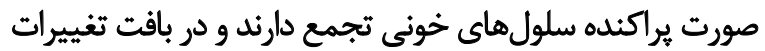

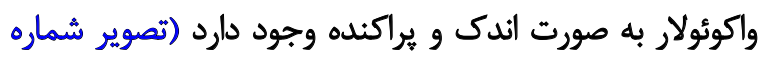

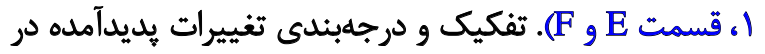

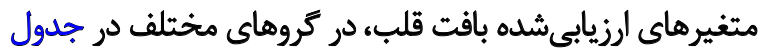
شماره r نشان داده شده است.

ثִ

نتايج تحقيق حاضر نشان داد در نمونههاى كروه كنترل

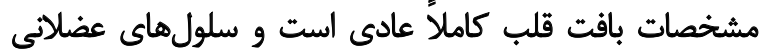

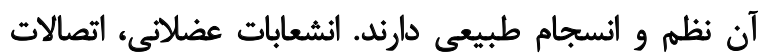

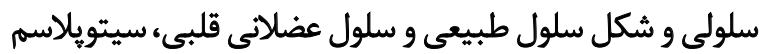

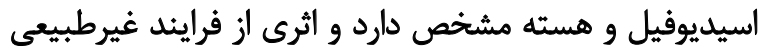

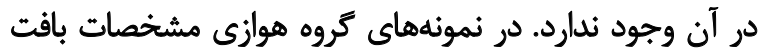

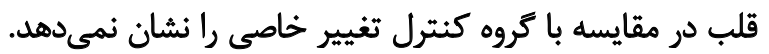

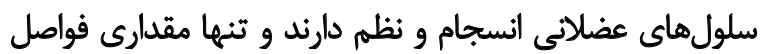

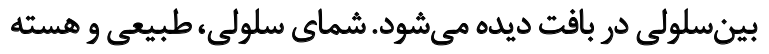

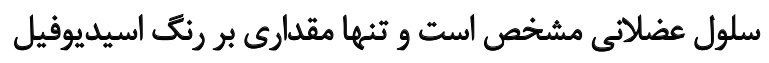

$$
\text { سيتويلاسم افزوده شده است. }
$$

در نمونههاى كروه بيولوريك به طور كل در بافت، اثرات نظم

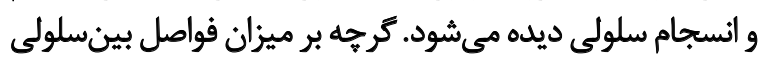

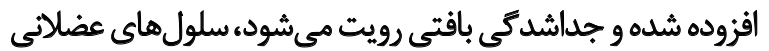

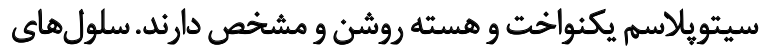

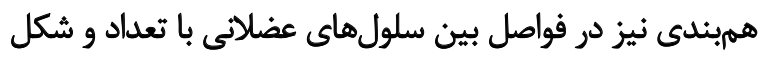

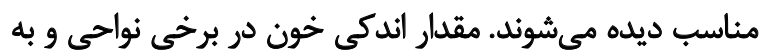

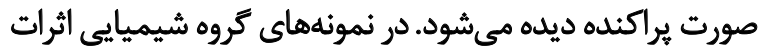




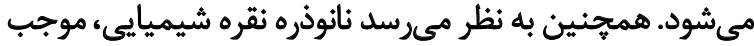

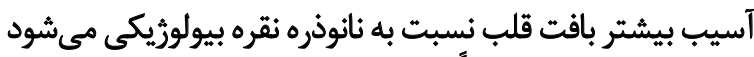

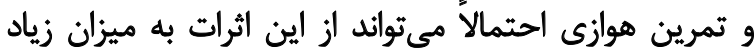

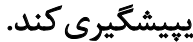

\section{مالاحظات اخلاقى}

\section{بيروى أو أصول الخالاث يُوهش}

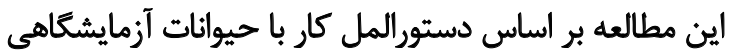

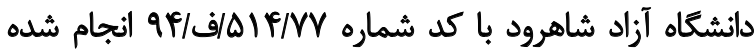

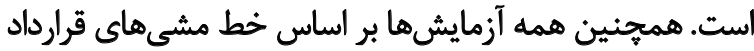

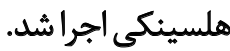

$$
\text { مامي مالى }
$$

اين تحقيق مستخرج از رساله دكتراى فروغ جواهرى هوشى،

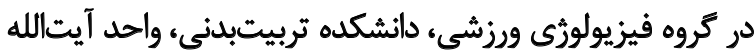

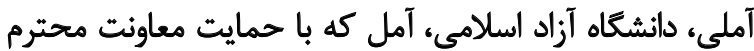
يُروهشى دانشكاه آزاد اسلامى واحد آيتا آلآله آملى آنجام شده

\section{مشار كت ثويسند مكان}

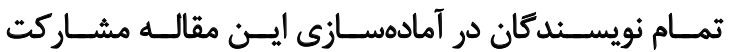
داشـــتهاند.

\section{تقارض مناقع}

طبق نظر نويسندكان هيجّونه تعارض منافعى در اين مقاله

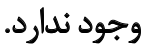

اين تأثير نانونقره شيميايي ممكن است به علت استفاده از عوامل

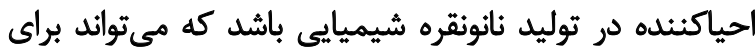

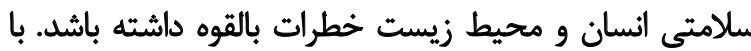

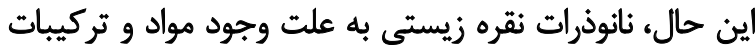

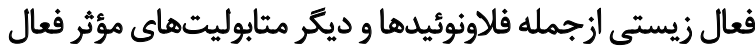

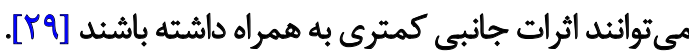

درمطالعه حاضر مشاهده شد بعد از • 1 هفته تمرين هوازي

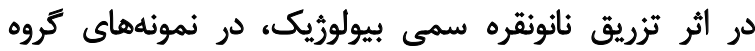

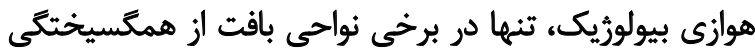

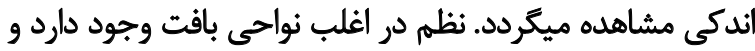



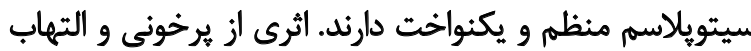

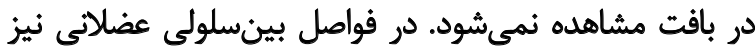

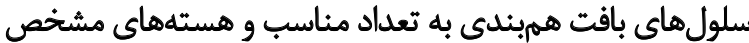

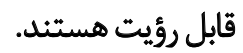

تنها در برخى نواحى بافت، ازهم بسيختگى اندى مشاهده

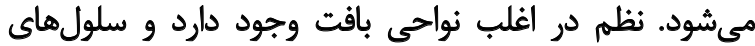

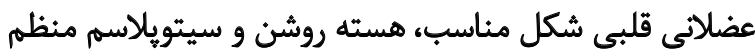

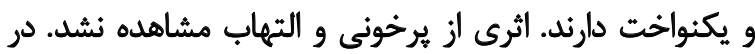

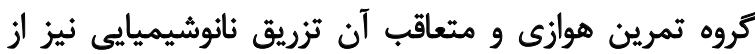

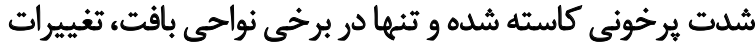

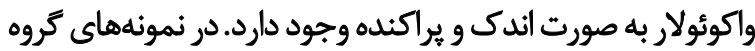

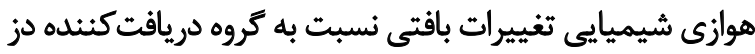

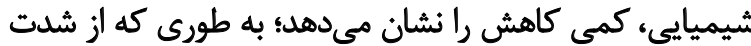

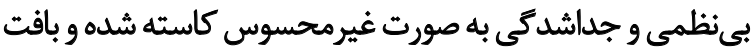
ستونها و ميدان هاى سلولى مشخصى بـ داردي

ازز ميزان يرخونى نيز كاسته شده است و تنها در برخى نواحى و

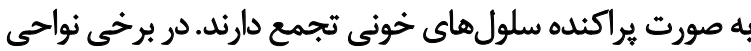

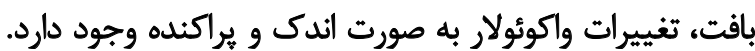

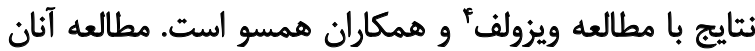

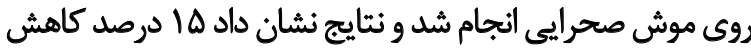

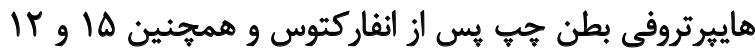

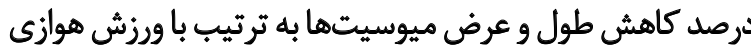



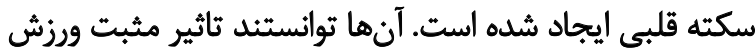

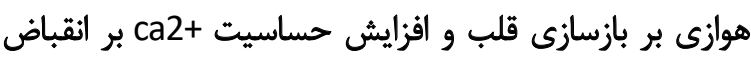
عضله قلب رانشان دهند [•r"].

$$
\text { تثيجهيرى }
$$

نتايج تحقيق نشان داد كه نانونقره بيولوريك و شيميايي موجب

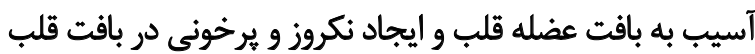




\section{References}

[1] Erb U, Aust KT, Palumbo G. Nanostructured materials: Processing, properties and potential applications. New York: Noyes Publications; 2002.

[2] Goddard WA, Brenner DW, Lyshevski SE, lafrate GJ. Handbook of nanoscience engineering, and technology. USA: CRC Press; 2002. [DOI:10.1201/9781420040623]

[3] Chen X, Schluesener HJ. Nanosilver:a nanoproduct in medical application. Toxicology Letters. 2008; 176(1):1-12. [DOI:10.1016/j.toxlet.2007.10.004] [PMID]

[4] Buzea C, Pacheco I, Robbie K. Nanomaterials and nanoparticles:Sources and toxicity. Biointerphases. 2007; 2(4):17-71. [DOI:10.1116/1.2815690]

[5] Gibbons B, Warner L. The role of antimicrobial silver nanotechnology. Medical Device and Diagnostic Industry Magazine. 2005; 27(5):164-9.

[6] Panacek A, Kvitek L, prucek R, Kolar M. et al Silver colloid nanoparticles:synthesis, characterization, and antibacterial activity. The Journal of Physical Chemistry. 2006; 110(33):16248-53. [DOI:10.1021/ jp063826h] [PMID]

[7] Tang J, Xi T. [Status of biological evaluation on silver nanoparticles(Chinese)]. Sheng Wu Yi Xue Gong Cheng Xue Za Zhi. 2008; 25(4):958-61. [PMD]

[8] Panyala NR, Pena-Mendez EM, Havel J. Silver or silver nanoparticles:A hazardous threat to the environment and human health? Journal of Applied Biomedicine. 2008; 6:117-29. [DOI:10.32725/jab.2008.015]

[9] Ostiguy C, Soucy B, Lapointe G, Woods C. Health Effects of Nanoparticles Second ed. Montreal: Institut de Recherche Robert-Sauvé en santé et en sécurité du travail; 2008

[10] Hamrahi-Michak M, Sadeghi SA, Haghighi $H$, Ghanbari-Kakavandi $Y_{\text {, }}$ Razavi-sheshdeh SA, Noughabi MT, et al. The toxicity effect of cerium oxide NP on blood cells of male rat. Annals of Biological Research. 2012; 3(6):2859-66

[11] Ranjbar Sardari RR, Rezaei Zarchi S, Talebi A, Nasri S. et al. Toxicological effects of silver nanoparticles in rats. African Journal of Microbiology Research. 2012; 6(27):5587-93. [DOI:10.5897/AJMR11.1070]

[12] Parka E, Bae E. Repeated-dose toxicity and inflammatory responses in mice by oral administration of silver nanoparticles. Environmental Toxicology and Pharmacology. 2010; 30(2):162-68. [DOI:10.1016/j. etap.2010.05.004] [PMID]

[13] Kim YS, Kim JS, Cho HS, Rha DS, et al. Twenty-eight-day oral toxicity, genotoicity, and gender-related tissue distribution of silver nanoparticles in Sprague-Dawley rats. Inhal. Toxicol. 2008; 20(6):575-83. [DOI:10.1080/08958370701874663] [PMID]

[14] Hyun JS, Lee BS, Ryu HY, Sung JH, et al. Effects of repeated silver nanoparticles exposure on the histological structure and mucins of nasal respiratory mucosa in rats. Toxicology Letters. 2008; 182(3):24-8. [DOI:10.1016/j.toxlet.2008.08.003] [PMID]

[15] Warren JS, Johnson KJ, Ward PA. Oxygen Radicals in Cell Injury and Cell Death. Pathology and Immunopathology Research. 1987; 6(6):301-15. [DOI:10.1159/000157061] [PMID]

[16] Sikka SC, Rajasekaran M, Hellstrom WJ. Role of oxidative stress and antioxidants in male infertility. Journal of Andrology. 1995; 16(6):464-8.

[17] Agarwal A, Saleh RA, Bedaiwy MA. Role of reactive oxygen species in the pathophysiology of humn reproduction. Fertility and Sterility. 2003; 79(4):829-43. [DOI:10.1016/S0015-0282(02)04948-8]
[18] Ochsendorf FR. Infection in male genital tract and reactive oxygen species. Human Reproduction Update. 1999; 5(5):399-420. [DOI:10.1093/ humupd/5.5.399] [PMID]

[19] Ahn J, Kim J. Mechanisms and consequences of inflammatory signaling in the myocardium. Current Hypertension Reports. 2012; 14(6):51016. [DOI:10.1007/s11906-012-0309-0] [PMID]

[20] Almofti MR, Ichikawa T, Yamashita K, Terada H, Shinohara Y. Silver ion induces a cyclosporine a-insensitive permeability transition in rat liver mitochondria and release of apoptogenic cytochrome c. Journal of Biochemistry. 2003; 134(1):43-9. [DOI:10.1093/jb/mvg111] [PMID]

[21] Vasili A, Sharifi G, Faramarzi M, Noori A, Yazdanshenas S. The effect of aerobic exercise on hepatotoxicity induced by intratracheal instillation of iron oxide nanoparticles in Wistar rats. General Physiology and Biophysics. 2016; 35(1):35-43. [DOI: 10.4149/gpb_2015031] [PMID]

[22] Shin SH, Ye MK. The effect of nano-silver on allergic rhinitis model in mice. Clinical and Experimental Otorhinolaryngology. 2012; 5(4):222-7. [DOI:10.3342/ceo.2012.5.4.222] [PMID] [PMCID]

[23] Wang Z, Xia T, Liu S. Mechanisms of nanosilver-induced toxicological effects:more attention should be paid to its sublethal effects. Nanoscale. 2015; 7(17):7470-81. [DOI:10.1039/C5NR01133G] [PMID] [PMCID]

[24] Lee JH, Kim YS, Song KS, Ryu HR, Sung JH, Park JD, et al. Biopersistence of silver nanoparticles in tissues from Sprague-Dawley Rats. Particle and Fibre Toxicology. 2013; 10(1):36-47.[DOI:10.1186/1743-8977-10-36] [PMID] [PMCID]

[25] Mogharnasi M, Baya J, Foadoddini M, Salehikia A, Hosseini M, Shahamat Nashtifani F. [The effect of colostrum along with aerobic and anaerobic exercise on lipid peroxidation and total antioxidant capacity of male wistar rats (Persian)]. Armaghane danesh Journal. 2016; 21(3):265-77.

[26] Pourali P, Yahyaei B. Biological production of silver nanoparticles by soil isolated bacteria and preliminary study of their cytotoxicity and cutaneous wound healing efficiency in rat. Journal of Trace Elements in Medicine and Biology. 2016; 34:22-31. [DOI:10.1016/j.jtemb.2015.11.004] [PMID]

[27] Naghsh N, Mashayekh A, Khodadadi S. [Effects of silver nanoparticle on lactate dehydrogenase activity and histological changes of heart tissue in male wistar rats (Persian)]. Journal of Fasa University of Medical Sciences/ Winter 2012; 2(4):303-7.

[28] Akradi L, Sohrabi Haghdoost I, Djeddi AN. Histopathologic and apoptotic effect of nanosilver in liver of broiler chickens. African Journal of Biotechnology. 2012; 11(22):6207-11. [DOI:10.5897/AJB11.1768]

[29] Martirosyan, A. Bazes, Y. Schneider. Nanotoxicology, 2014; 8(5):57382. [DOI:10.3109/17435390.2013.812258] [PMID]

[30] Wisløff U, Loennechen JP, Currie S, Smith GL, Ellingsen $\varnothing$. Aerobic exercise reduces cardiomyocyte hypertrophy and increases contractility, $\mathrm{Ca}^{2+}$ sensitivity and SERCA-2 in rat after myocardial infarction. Cardiovascular Research. 2002; 54(1):162-74. [DOI:10.1016/S0008-6363(01)00565-X] 